\title{
España ante la debida diligencia en violencia de género
}

\author{
Spain and the due diligence in gender violence
}

\author{
Ana Manero SALVAdor \\ Profesora Titular de Derecho Internacional Público \\ y Relaciones Internacionales \\ (acreditada como Catedrática) \\ Universidad Carlos III de Madrid \\ Ana.manero@uczm.es
}

Resumen: Uno de los problemas más graves de derechos humanos en España es la violencia de género, especialmente la ejercida por actores no estatales y que tiene lugar en el ámbito privado o familiar. Los Estados tienen el deber de cumplir con el estándar de debida diligencia, por lo que este trabajo tiene como finalidad determinar en qué consiste este estándar y examinar si España lo cumple teniendo en cuenta las obligaciones establecidas en el marco universal de protección de los derechos humanos.

Palabras clave: violencia de género, debida diligencia, España, CEDAW

\begin{abstract}
One of the most serious human rights problems in Spain is gender violence, especially that carried out by non-state actors and takes place in the private or family sphere. The States have the duty to comply with the due diligence standard. This paper has the aim of determining the elements of this standard and examining if Spain complies with it, according to UN developments.
\end{abstract}

Keywords: gender violence, due diligence, Spain, CEDAW

Sumario: 1. INTRODUCCIÓN. 2. LA DEBIDA DILIGENCIA Y LA VIOLENCIA DE GÉNERO. 2.1. Prevención. 2.2. Protección. 2.3. Investigación, juzgamiento y castigo. 2.4. Reparación. 3. ESPAÑA Y LA DEBIDA DILIGENCIA EN MATERIA DE VIOLENCIA CONTRA LAS MUJERES. 3.1. España y la debida diligencia sistémica. 3.2. España y la debida diligencia individual: el asunto Ángela González Carreño contra España. 4. REFLEXIÓN FINAL. 5. BIBLIOGRAFÍA

\section{INTRODUCCIÓN}

L

a violencia de género es una lacra que afecta a la Comunidad Internacional en su conjunto. De hecho, según ONU Mujeres, entre el $35 \%$ y el $70 \%$ de las mujeres del mundo han sufrido violencia sexual y/o física en su vida. No estamos hablando de un problema que afecte únicamente a los países en desarrollo, dado que se estima que el $43 \%$ de las mujeres de la Unión Europea han sufrido este tipo de violencia. $\mathrm{Y}$ es que es un tipo de violencia que da lugar a la muerte de mujeres, en tanto que se considera «que en prácticamente la mitad de los casos de mujeres víctimas de homicidios [...] el autor 
de la agresión fue un familiar o un compañero sentimental». ${ }^{1}$ Tanto desde los Estados como desde la Comunidad Internacional se han establecido instrumentos normativos y mecanismos que pretenden luchar contra la violencia que sufren las mujeres, si bien la desconfianza hacia las instituciones por parte de estas mujeres en situación de vulnerabilidad les hace renuentes a acudir a ellos. De hecho, menos del $40 \%$ de las mujeres que han experimentado violencia de género busca ayuda de algún tipo, y muchas de ellas lo hacen en su ámbito privado, acudiendo a amigas o familiares. Menos de un $10 \%$ acude a la policía $^{2}$. En muchas ocasiones las instituciones fallan a la hora de proteger a las mujeres víctimas de violencia de género, lo que da lugar a la responsabilidad internacional del Estado, como veremos.

Por lo que atañe a España, en 2017 han muerto 51 mujeres. De ellas, solo 12 habían denunciado a sus maltratadores ${ }^{3}$. A estas víctimas mortales deben sumarse los asesinatos de hijos, que se estiman en medio centenar en la última década ${ }^{4}$.

Desde el Derecho Internacional se considera que la violencia de género constituye una violación de los derechos humanos, tal y como se explicita por parte del Comité para la eliminación de todas las formas de discriminación contra la mujer (en adelante, Comité de la CEDAW), en su Observación General $\mathrm{n}^{\circ} 19$, en la que indica que «la violencia contra la mujer es una forma de discriminación que inhibe gravemente la capacidad de la mujer de gozar de derechos y libertades en pie de igualdad con el hombre» (párr. 1), es más, «la violencia contra la mujer, que menoscaba o anula el goce por la mujer de sus derechos humanos y libertades fundamentales en virtud del derecho internacional o de convenios específicos de derechos humanos, constituye discriminación» (párr. 7) y esta «discriminación no se limita a los actos cometidos por los gobiernos o en su nombre [...]. Los Estados Partes se comprometen

1 ONU Mujeres, Hechos y cifras: acabar con la violencia contra mujeres y niñas, ONU Mujeres, 2016, disponible en: http://www.unwomen.org/es/what-we-do/ending-violence-againstwomen/facts-and-figures [consultado: 13/05/2018].

2 United Nations Statistics Division, «Chapter 6. Violence against Women», The World's Women 2015, Naciones Unidas, 2015, p. 159, disponible en https://unstats.un.org/unsd/gender/worldswomen.html [consultado: 13/05/2018].

3 Víctimas mortales por violencia de género. Ficha resumen-Datos provisionales a 2 de noviembre de 2017, Ministerio de Sanidad, Servicios Sociales e Igualdad, disponible en http://www. violenciagenero.msssi.gob.es/violenciaEnCifras/victimasMortales/fichaMujeres/pdf/VMortales_2017_11_02.pdf [consultado: 09/05/2018].

$4 \ll \mathrm{El}$ asesinato de hijos también es violencia de género», en Europa Press, 3 de agosto de 2015. 
a adoptar todas las medidas adecuadas para eliminar la discriminación contra la mujer practicada por cualesquier personas, organizaciones o empresas. En virtud del derecho internacional [...] los Estados también pueden ser responsables de actos privados si no adoptan medidas con la diligencia debida para impedir la violación de los derechos o para investigar y castigar los actos de violencia y proporcionar indemnización» (párr. 9) ${ }^{5}$.

Teniendo en cuenta que el Comité de la CEDAW es el órgano encargado de interpretar la Convención para la eliminación de todas las formas de discriminación contra la mujer (en adelante, CEDAW), se puede deducir que desde este instrumento se obliga a los Estados partes a actuar con debida diligencia en el ámbito de la violencia contra la mujer, pero ¿en qué consiste esta debida diligencia? A ello se dedicarán las siguientes páginas, para más adelante analizar si España, con un grave problema de derechos humanos en este ámbito, respeta sus obligaciones internacionales.

\section{LA DEBIDA DILIGENCIA Y LA VIOLENCIA DE GÉNERO}

El concepto de debida diligencia a la hora de determinar la responsabilidad internacional del Estado por actos de agentes no estatales se estableció por primera vez por la Corte Interamericana de Derechos Humanos en el asunto Velásquez Rodríguez contra Honduras ${ }^{6}$, afirmando que el Estado era responsable por la falta de debida diligencia a la hora de prevenir violaciones de derechos humanos, por lo que se considera que el Estado tiene la obligación de ejercer la debida diligencia para asegurar el cumplimiento de sus obligaciones relativas a la protección de los derechos humanos. Este testigo lo recoge el artículo 4.c) de la Declaración sobre la eliminación de la violencia contra la mujer, que afirma que los Estados deben «proceder con la debida diligencia a fin de prevenir, investigar, y, conforme a la legislación nacional, castigar todo acto de violencia contra la mujer, ya se trate de actos perpetrados por el Estado o por particulares», siguiendo, pues, la línea establecida en la Observación General n $19^{7}$.

5 CEDAW, Recomendación General 19. La violencia contra la mujer, de 29 de enero de 1992.

6 Asunto Velásquez Rodríguez contra Honduras, Corte Interamericana de Derechos Humanos, 1988, Ser. C, nº4, párr. 172.

7 QuRESHI, S., «The emergence/extention of Due Diligence Standard to Assess the State Response towards Violence against Women/Domestic Violence», South Asian Studies, vol. 28, 2013 , p. 55 y ss. 
La atribución de responsabilidad al Estado por actos de agentes estatales no va a ser objeto de análisis en este trabajo, dada la gravedad de la violencia contra las mujeres ejercida en el ámbito doméstico en España, cuestión sobre la que versará este trabajo, por lo que el estándar de debida diligencia va a ser clave para determinar si España cumple o no con sus obligaciones internacionales, y es que mediante el estándar de debida diligencia, será posible determinar la responsabilidad internacional del Estado también por omisiones, tal y como se refleja en varios los instrumentos que aluden a la debida diligencia en relación a la violencia de género ${ }^{8}$. Asimismo, desde 2006, puede considerarse que estamos ante una obligación de naturaleza consuetudinaria ${ }^{9}$, además de convencional regional ${ }^{10}$. Si bien se comienza a perfilar en el ámbito regional interamericano y empieza aplicarse en relación a la violencia contra la mujer en esta región ${ }^{11}$, su testigo es recogido por el Tribunal Europeo de Derechos Humanos ${ }^{12}$, lo que reflejará en el Convenio del Consejo de Europa sobre prevención y lucha contra la violencia contra la mujer y la violencia

8 Vid. Informe de la Cuarta Conferencia Internacional de las Mujeres de Beijing, 4-15 septiembre de 1995, Declaración y Plataforma de Acción de Beijing, A/CONF.177/20/Rev.1, párr. 124.b, Convención Interamericana para prevenir, sancionar y erradicar la violencia contra las mujeres de 1994 (art. 7.b), Protocolo a la Carta Africana de los Derechos Humanos y de los Pueblos sobre los derechos de las mujeres en África (art. 4), Convenio del Consejo de Europa sobre prevención y lucha contra la violencia contra la mujer y la violencia doméstica del Consejo de Europa (art. 5), Directiva 2012/29/UE por la que se establecen normas mínimas sobre los derechos, el apoyo y la protección de las víctimas de delitos, y por la que se sustituye la Decisión marco 2001/220/JAI del Consejo (DO L 315/57 de 14.11.2012), considerandos 5, 6, 17 y 18.

9 «Tomando como base la práctica y la opinio iuris [...] se puede concluir que hay una norma del derecho internacional consuetudinario que obliga a los Estados a prevenir y responder con la debida diligencia a los actos de violencia contra la mujer». Integración de los derechos humanos de la mujer y perspectiva de género: violencia contra la mujer. La norma de la debida diligencia como instrumento para la eliminación de la violencia contra la mujer, Informe de la Relatora sobre la violencia contra la mujer, sus causas y consecuencias, Yakin Ertürk, E/CN.4/2006/61 de 20 de enero de 2006, párr. 29, y 19 y ss.

Asimismo, vid. Hasselbacher, L., «State Obligations Regarding Domestic Violence: The European Court of Human Rights, Due Diligence, and International Legal Minimus of Protection», Northwestern Fournal of International Human Rights, vol. 8, 2010, p. 200; y Nota del Secretario General: La violencia contra la mujer, sus causas y sus consecuencias, A/69/38 de 1 de septiembre de 2014, párr. 49 y 56 y ss.

10 Vid. nota al pie 8.

11 Caso Maria da Penha Maia Fernandes contra Brasil, Comisión Interamericana de Derechos Humanos, Indorme 54/01 de 16 de abril de 2001, párr. 56.

12 Z. y otros contra Reino Unidos, demanda 29392/95 (2001), Comisión Europea de Derechos Humanos, y E. y otros contra Reino Unido, demanda 33218/96 (2002), Tribunal Europeo de Derechos Humanos. 
doméstica, más conocido como Convenio de Estambul, y por el Comité de la $\mathrm{CEDAW}^{13}$.

¿Cuál es el contenido de esta obligación? Al hablar de la debida diligencia, según la Relatora Majoo, hay que distinguir dos tipos: la diligencia debida individual y la diligencia debida sistémica. La primera «se refiere a las obligaciones que tienen los Estados hacia ciertos individuos, o grupos de individuos, de prevenir, proteger, castigar y proporcionar recursos efectivos para cada caso» ${ }^{14}$. Esta modalidad «requiere flexibilidad, ya que los procedimientos adoptados [...] deben reflejar las necesidades y preferencias de los individuos afectados [...] [y] también exige a los Estados que castiguen no solo a los autores de delitos, sino también a aquellos que no cumplan con su deber de responder ante la comisión de violaciones $\gg^{15}$. Por otro lado, la diligencia debida sistémica «se refiere a las obligaciones de los Estados de asegurar un modelo integral y sostenido de prevención, protección, castigo y reparación de los actos de violencia contra la mujer. A un nivel sistémico, los Estados pueden cumplir su responsabilidad de proteger, prevenir y castigar, mediante, entre otras cosas, la adopción o modificación de leyes; la elaboración de estrategias, planes de acción y campañas de sensibilización, y la prestación de servicios; el refuerzo de la capacidad y facultad de la policía, fiscales y jueces; la asignación de los recursos necesarios para las iniciativas de cambios transformadores; y la rendición de cuentas por parte de aquellos que no aseguren la protección y prevención y de quienes vulneren los derechos humanos de la mujer. Asimismo, los Estados tienen que participar de forma más concreta en la transformación de la sociedad en general para combatir la desigualdad de género y la discriminación estructurales y sistémicas ${ }^{16}$.

De acuerdo con lo dicho, varias son las manifestaciones de esta obligación. En primer lugar, se establece el deber de prevención, para evitar la violación. Esta es la única medida de carácter previo, dado que, a continuación, los siguientes deberes son posteriores al ejercicio de la violencia, como son la protección, la investigación, enjuiciamiento y castigo y, por último, el remedio.

13 Comunicación 2/2003, AT. Contra Hungría (2005) de 26 de enero.

14 Énfasis añadido.

15 Informe de la Relatora Especial sobre la violencia contra la mujer, sus causas y consecuencias, Rashida Majoo, A/HRC/23/49 de 14 de mayo de 2013, párr. 70.

16 Id., párr. 71.

Vid. FARRIOR, S., «The Due Diligence Standard and Violence against Women», Interights Bulletin, $\mathrm{n}^{\circ} 14,2004$, p. 150 y 151. 


\subsection{Prevención}

El Manual de Legislación sobre la violencia contra la mujer recomienda que la prevención incluya medidas de concienciación sobre los derechos de la mujer e igualdad, que los planes de estudio sirvan para modificar patrones de conducta discriminatorios y estereotipados y que los medios de comunicación sensibilicen contra la violencia de género ${ }^{17}$.

Más concretamente, al hablar del deber de prevención, en mi opinión, se debe distinguir si estamos ante situaciones en las que el Estado debe satisfacer el estándar de debida diligencia individual o sistemática, de conformidad con lo indicado por la Corte Interamericana de Derechos Humanos en la sentencia Campo Algodonero donde se afirma que «los Estados deben adoptar medidas integrales para cumplir con la debida diligencia en casos de violencia contra las mujeres. En particular, deben contar con un adecuado marco jurídico de protección, con una aplicación efectiva del mismo y con políticas de prevención y prácticas que permitan actuar de una manera eficaz ante las denuncias. Asimismo, los Estados deben adoptar medidas preventivas en casos específicos de violencia» ${ }^{18}$.

La prevención individual se refiere a una situación concreta y particularizada en la que el Estado ha tenido conocimiento de una situación de violencia contra la mujer. En esta situación, la actuación rápida y decidida por parte de los Estados, enmarcada en una perspectiva de género, es esencial. Para determinar cómo deber ser esta respuesta, considero especialmente meticulosa la labor desarrollada por la Corte Interamericana de Derechos Humanos, a la hora de determinar en qué consiste el deber de prevención y cómo podemos considerar que el Estado ha actuado con debida diligencia:

i) El Estado «debe tener conocimiento de una situación de riesgo real e inmediato»;

ii) Ese riesgo debe amenazar a «un individuo o grupo de individuos determinados o determinables»;

iii) «se precisa verificar posibilidades razonables de prevenir o evitar ese riesgo» ${ }^{19}$.

17 Departamento de Asuntos Económicos y Sociales, División para el Adelanto de la Mujer de las Naciones Unidas, Manual de Legislación sobre la violencia contra la mujer, N.U., Nueva York, 2010, p. 29.

18 Asunto González y otras contra México (Campo Algodonero), Excepción preliminar, Fondo, Reparaciones y Costas, Corte Interamericana de Derechos Humanos, Serie C, $\mathrm{n}^{\circ}$ 2015, 2009, párr. 258.

19 MartíneZ Vargas, J.R.; Vega Barbosa, G., «La obligación estatal de prevención a la luz del corpus iuris internacional de protección de la mujer contra la violencia de género», Revista Ius et Praxis, $\mathrm{n}^{\circ} 2,2013$, p. 350. 
Ahora bien, en mi opinión, este estándar de prevención se presenta como bastante restrictivo y se enmarca en una situación que, en mi opinión, puede calificarse de violación generalizada y sistemática de los derechos de las mujeres, como es la situación de Ciudad Juárez, donde la inacción por parte de los Estados en relación a la violencia contra la mujer está plagada de los más perniciosos estereotipos, como se pone de manifiesto en la sentencia de Campo Algodonero, en la que, una vez denunciada la desaparición de sus hijas, la policía despreció a las madres y su denuncia, aduciendo que las niñas estarían con sus novios y que «si le pasaba eso era porque ella se lo buscaba, porque una niña buena, una mujer buena, está en casa $\gg^{20}$, lo que es especialmente doloroso y genera una enorme impotencia en un entorno tan violento para las mujeres y niñas como es esa ciudad fronteriza. Ahora bien, este estándar de prevención no es extrapolable a otras realidades geográficas, por lo que, en todo caso, a la hora de determinar el deber de prevención es esencial determinar el contexto en el que la violencia se produce.

Más flexible es la doctrina del riesgo, formulada por Abravamovich en función de cuatro elementos:

i) «Que exista una situación de riesgo real o inmediato que amenace derechos y que surja de la acción o las prácticas particulares, esto es, se requiere que el riesgo no sea meramente hipotético o eventual y, además, que no sea remoto, sino que tenga posibilidad cierta de materializarse en lo inmediato.

ii) Que la situación de riesgo amenace a un individuo o a un grupo determinado, esto es, que exista un riesgo particularizado. Lo anterior supone un requisito más estricto que la sola existencia de un riesgo general o una situación extendida de inseguridad que afecta a una situación extendida de inseguridad que afecta al conjunto de la comunidad.

iii) Que el Estado conozca el riesgo o hubiera debido razonablemente conocerlo o preverlo. En tal sentido, aquí cuenta tanto la evidencia que determina que las agencias habían obtenido información sobre la situación de riesgo, como también la previsibilidad del riesgo, esto es, la posibilidad de establecer cierta presunción de conocimiento de este riesgo a partir de las circunstancias del caso [...]. Así, los Estados

20 Asunto González y otras contra México (Campo Algodonero), Excepción preliminar, Fondo, Reparaciones y Costas, Corte Interamericana de Derechos Humanos, Serie C, $\mathrm{n}^{\circ}$ 2015, 2009, párr. 63. 
tienen el deber [...] de producir información y hacer seguimiento de la situación de violencia que sufren algunos grupos sociales y sectores de la población, de modo que no puede admitirse como excusa el desconocimiento de situaciones de violencia en esos casos. Además, existen riesgos que son previsibles por su envergadura, por su extensión en el tiempo, porque obedecen a prácticas o patrones sistémicos que hacen imposible su desconocimiento por parte de la autoridad estatal.

iv) Finalmente, que el Estado pueda razonablemente prevenir o evitar la materialización del riesgo. Para poder imputar responsabilidad se requiere entonces, primero, que el riesgo sea, por sus características, evitable y que el Estado esté en condiciones de adoptar medidas capaces de paliar la situación y evitar la materialización del riesgo. Este último elemento se refiere tanto a las características de los factores de riesgo que se presentan como a las capacidades operativas de los agentes públicos que podían actuar en ese escenario determinado $»^{21}$.

No parece difícil conocer cuándo un Estado ha actuado con debida diligencia en este ámbito. Las autoridades policiales, judiciales y gubernativas deben tomar como prioridad absoluta la protección de la mujer y evitar que el daño se produzca. En este sentido, se señala que estamos ante una obligación de comportamiento, no de resultado, «pero es probable que una ausencia de comportamiento constituya una ausencia de resultados $\gg^{22}$, que den lugar a una violación de derechos humanos y a la responsabilidad internacional del Estado.

Por lo que atañe a la prevención en el marco de la debida diligencia sistémica, la forma más habitual en la que los Estados la han acometido, ha sido a través de medidas legislativas, campañas de sensibilización y capacitación de

21 AвRAmovich, V., «Responsabilidad estatal por violencia de género: comentarios sobre el caso «Campo Algodonero» en la Corte Interamericana de Derechos Humanos», Anuario de Derechos Humanos, 2010, p. 174.

22 Informe de la Relatora Especial sobre la violencia contra la mujer, sus causas y consecuencias, Rashida Manjoo, A/HRC/23/49 de 14 de mayo de 2013, párr. 16 y 72.

Asimismo, Goldscheid y Liebowitz señalan: «scholars generally agree that the due diligence obligation is one of means and not results. In other words, an act of domestic violence itself would not be evidence of State failure to exercise due diligence. It is rather «a lack of reasonableness in measures of prevention and/or a lack of seriousness in measures of responsse that indicate such a failure. This does not mean, however, that a State can simply claim that it did not have the structures in place to prevent or redress acts of gender violence. This defense fails because acting with due diligence requires the State to take measures that «have a real prospect of altering the outcome or mitigating the harm». GoldSCHEID, J.; LiEBOWITZ, D.J., «Due Diligence and Gender Violence: Parsing its Power and its Perils», Cornell International Law fournal, n 301, 2015, p. 310. 
profesionales ${ }^{23}$. Se puede considerar que la prevención debe incluir «apoyo social estructural como educación, empleo y vivienda; [...] abordar cuestiones intersectoriales y factores de riesgo que perpetúan la violencia, como la desigualdad de género, los estereotipos y las percepciones culturales de las mujeres, la pobreza, la educación de las mujeres y la independencia económica y [...] formular e implementar leyes que no sólo aborden las formas específicas de la violencia contra las mujeres, sino también las causas subyacentes que la provocan $\gg^{24}$. Pero, en definitiva, requiere de medidas que vayan a la raíz, al origen del problema, al vínculo entre violencia de género y la discriminación contra la mujer, insistiendo en la perpetuación de los patrones socio-culturales, en el combate de los estereotipos de género y las desigualdades. La necesidad de prestar atención a las causas últimas de la violencia contra las mujeres debe traer consigo un análisis de la vinculación de ésta con otras formas de discriminación y requiere la necesidad de atender a las múltiples e intersectoriales formas de discriminación, como herramienta de cambio cultural que acabe con la normalización de la justificación de la violencia de género ${ }^{25}$.

\subsection{Protección}

Una vez que la violencia ha tenido lugar, los Estados debe proceder a proteger a la víctima, con la finalidad de «evitar la recurrencia de nuevos actos de violencia y asegurar que las víctimas/sobrevivientes reciban servicios adecuados y oportunos» ${ }^{26}$. En este sentido, la legislación debe crear servicios de apoyo amplios e integrados, que presten un apoyo suficiente a las víctimas, que su ubicación permita un acceso equitativo a los servicios y establecer lo que se conoce como el «nivel mínimo de servicios de apoyo», que incluya una línea telefónica nacional, una casa de acogida por cada 10.000 habitantes, donde además de alo-

23 Informe de la Relatora Especial sobre la violencia contra la mujer, sus causas y consecuencias, Yakin Ertük, Integración de los derechos humanos de la mujer y perspectiva de género: violencia contra la mujer. La norma de debida diligencia como instrumento para la eliminación de la violencia contra la mujer, E/CN.4/2006/62 de 20 de enero de 2006, párr. 38.

24 Abdul Aziz, Z.; Moussa, J. (dir.), Marco de la Debida Diligencia. Marco sobre responsabilidad estatal para la eliminación de la violencia contra las mujeres, Iniciativa Internacional de Derechos Humanos, 2016, p. 15.

25 Goldscheid, J.; Liebowitz, D.J., «Due Diligence and Gender Violence: Parsing its Power and its Perisl», op. cit., p. 309.

26 Abdul AzIz, Z.; Moussa, J. (dir.), Marco de la Debida Diligencia..., op. cit., p. 36. 
jamiento seguro de emergencia se provea asesoramiento y asistencia, un centro de defensa y asesoramiento por cada 50.000 mujeres, un centro de crisis para casos de violación por cada 200.000 mujeres, y acceso a la asistencia sanitaria, incluyendo salud reproductiva y profilaxis contra el VIH/SIDA ${ }^{27}$.

En su informe, la Relatora Ertürk, señala que los Estados han implementado diversas medidas para cumplir con la obligación de debida diligencia en cuanto a la protección «que consisten principalmente en la provisión de servicios para la mujer, por ejemplo, líneas telefónicas directas, atención de salud, centros de asesoramiento, asistencia letrada, centros de acogida, órdenes de interdicción y ayuda financiera para las víctimas de violencia $\gg^{28}$. Ahora bien, la Relatora critica la debilidad de estas medidas, que en muchos casos no cuentan con bases jurídicas en los ordenamientos internos, la falta de formación de los jueces sobre la materia o la policía, las insuficiencias de los servicios, o el enfoque, centrado en la ayuda de urgencia a corto plazo y no en dotar a las víctimas de medios para que superen su situación ${ }^{29}$. En esta línea indican Abdul Aziz y Moussa que los Estados deben poner a disposición medios y que estos sean accesibles y estén coordinados. La disponibilidad se refiere a que, a ser posible, se cuente con centros de atención integrados, donde los servicios se presten en un único lugar, con personal capacitado en violencia de género, y en el que reciban asesoramiento, tratamiento, que cuenten con centros de apoyo y de refugio, políticas a medio y largo plazo que permitan a las mujeres acceder a una vivienda y a la independencia económica. Además, estos servicios deben ser accesibles, tanto a nivel económico como físico (transporte y lugar), lo que constituye un reto muy importante en zonas rurales; y en los que la información sea fácilmente aprehensible. Asimismo, estos servicios deben atender a las necesidades de los colectivos de mujeres especialmente vulnerables, como migrantes, LGTBI, refugiadas, y pertenecientes a minorías étnicas, entre otras ${ }^{30}$. Asimismo, las órdenes de protección deben estar disponibles y se debe garantizar el acceso a ellas, como primer paso para vivir libres de violencia. De hecho «es fundamental que [las normas que regulen el acceso a estas órdenes]

27 Departamento de Asuntos Económicos y Sociales, División para el Adelanto de la Mujer de las Naciones Unidas, Manual de Legislación sobre la violencia contra la mujer, Naciones Unidas, op. cit., p. 32.

28 Integración de los derechos humanos de la mujer y perspectiva de género: violencia contra la mujer. La norma de la debida diligencia como instrumento para la eliminación de la violencia contra la mujer, Informe de la Relatora sobre la violencia contra la mujer, sus causas y consecuencias, Yakin Ertürk, E/CN.4/2006/61 de 20 de enero de 2006, párr. 47.

29 Id., párr. 48 y 49.

30 Abdul AzIz, Z.; Moussa, J. (dir.), Marco de la Debida Diligencia..., op. cit., p. 36 y ss. 
definan ampliamente la violencia contra las mujeres e incluyan la disponibilidad de las órdenes de protección en los casos de violencia doméstica. [...] Para ser efectivas, [...] deben permitir que las víctimas continúen en sus hogares [...], y en sus lugares de trabajo para permitirles continuar con su vida diaria», y deben atender a los retos que supone el acoso cibernético ${ }^{31}$.

Por otro lado, el primer ámbito en el que se activa la función de protección tiene lugar en los servicios de emergencia, fundamentalmente en la actuación policial, que debería actuar con rapidez para evitar un daño mayor. La capacitación de la policía es, pues, esencial, y, de conformidad con la Resolución de la Asamblea General 65/228 de 31 de marzo de 2011, Fortalecimiento de las respuestas en materia de prevención del delito y justicia penal a la violencia contra la mujer, en el Anexo donde figuran las Estrategias y Medidas Prácticas Modelo Actualizadas para la eliminación de la violencia contra la mujer en el campo de la prevención del delito y la justicia penal, se insta a los Estados a que se «promuevan la utilización de los conocimientos especializados de la policía, los fiscales y otros funcionarios del sistema de justicia penal, en especial mediante el establecimiento, de ser posible, de unidades especializadas o de personal y tribunales especializados, o mediante la reserva de tiempo en los tribunales, y velen por que todos los agentes de policía, fiscales y otros funcionarios del sistema de justicia penal reciban de forma periódica e institucional capacitación que los sensibilice frente a las cuestiones de género y la infancia y aumenten su capacidad para combatir la violencia contra la mujer $\gg^{32}$.

\subsection{Investigación, juzgamiento y castigo}

La Relatora Ertürk considera que esta obligación «ha sido considerada generalmente por los Estados como la obligación de aprobar o modificar leyes y reforzar la capacidad y las competencias de la policía, los fiscales y los magistrados» ${ }^{33}$. Estamos, ante un factor esencial, dado el importante grado de

31 Id., p. 46.

32 Resolución de la Asamblea General 65/228 de 31 de marzo de 2011, Fortalecimiento de las respuestas en materia de prevención del delito y justicia penal a la violencia contra la mujer, Anexo, párr. 16.c).

33 Integración de los derechos humanos de la mujer y perspectiva de género: violencia contra la mujer. La norma de la debida diligencia como instrumento para la eliminación de la violencia contra la mujer, Informe de la Relatora sobre la violencia contra la mujer, sus causas y consecuencias, Yakin Ertürk, E/CN.4/2006/61 de 20 de enero de 2006, párr. 50. 
impunidad de la violencia contra las mujeres. El miedo al estigma, al rechazo, la sensación de impotencia o vulnerabilidad de las víctimas, tienen como consecuencia que estemos ante un delito que no se denuncia, y si se hace es de manera mínima ${ }^{34}$, por ello, es esencial desarrollar políticas que tengan por objeto reducir el desistimiento ${ }^{35}$, creando un clima de confianza en el que la investigación y el juzgamiento puedan realizarse.

En este punto se deben diferenciar tres fases. La primer de ellas, la investigación, compete a funcionarios policiales y del ministerio público una vez que han tenido conocimiento de la comisión del ilícito. Los primeros deben responder «con diligencia» todas y cada una de las solicitudes de ayuda y protección, llegando incluso a considerarse el establecimiento de una obligación positiva de investigar, de manera efectiva, rápida, imparcial y exhaustiva ${ }^{36}$, hasta en el caso de que la denunciante no sea la víctima, la atención a las llamadas de asistencia de la violencia de género debe tener la misma prioridad que la dada a otros tipos de violencia, y, una vez que reciban una denuncia deben llevar a cabo una «evaluación coordinada de riesgos», «interrogando a las partes y los testigos [...]; tomando nota de la denuncia en detalle; asesorando a la demandante sobre sus derechos; cumplimentando y presentando un informe oficial sobre la denuncia; proporcionando u organizando el transporte de la demandante al hospital o centro médico, en caso de necesidad o solicitud; proporcionando u organizando el transporte de la demandante y sus hijos o dependientes» y proporcionando protección ${ }^{37}$. En mi opinión, la capacitación de la policía, como primer paso para garantizar un inicio adecuado de la investigación y continuar hacia en enjuiciamiento y posterior castigo, es esencial. El relato de la Relatora al narrar cómo en diferentes misiones las mujeres informaban de cómo las autoridades las desalentaban, llegando incluso a intimidarlas para evitar que presentaran una denuncia, los intentos de reconciliar

$34 \ll$ Por diversas razones, a menudo las víctimas no denuncian [...]. Varias encuestas realizadas en los países europeos revelaron que las tasas de denuncia varían entre el 2-10\% en Finlandia y el $25 \%$ en Dinamarca. Las investigaciones estiman que del 17 al 35\% de las mujeres colombianas han sido víctimas de violencia sexual, sin embargo, casi el $90 \%$ de éstas no lo denunciaron. Un estudio en Guatemala estima que más del $75 \%$ de los delitos en razón de género no se denuncian debido al estigma que acompaña a las mujeres víctimas y a los estereotipos sexistas». ABDUL AzIZ, Z.; Moussa, J. (dir.), Marco de la Debida Diligencia..., op. cit., p. 55.

35 Id., p. 60.

36 Id., p. 63.

37 Departamento de Asuntos Económicos y Sociales, División para el Adelanto de la Mujer de las Naciones Unidas, Manual de Legislación sobre la violencia contra la mujer, op. cit., p. 37. 
o de establecer procedimientos de mediación, condenan a la víctima a serlo en el largo plazo. De hecho, la Relatora afirma que «el incumplimiento por parte de las autoridades policiales al no investigar en detalle los delitos de violencia contra la mujer es un hecho frecuente. Incluso en los casos de violencia contra la mujer que llegan al sistema judicial, existe todavía un número preocupante de ejemplos de jueces que dictan sentencias leves o insuficientes por esos delitos» ${ }^{38}$.

Por su parte, los fiscales, deben contar con normas que dispongan que la responsabilidad de iniciar la investigación recae sobre ellos y no sobre las demandantes, independientemente del nivel de violencia ejercida. Asimismo, deben informar a las víctimas de «sus derechos, de los detalles del proceso, de los servicios, mecanismos de apoyo y medidas de protección sostenibles, de las oportunidades de obtener restitución y compensación a través del sistema judicial, de los detalles de los actos relacionados con su caso, incluidos el lugar y la hora de las audiencias» ${ }^{39}$, entre otras.

Finalmente, se recomienda que existan políticas favorables a la detención $\mathrm{y}$ al enjuiciamiento del delito, al considerar que existen razones fundadas de la comisión de un ilícito ${ }^{40}$.

La segunda fase sería la del juzgamiento, más concretamente, en el ejercicio de la jurisdicción penal contra los responsables de la comisión del ilícito. El procedimiento debe ser oportuno y acelerado, a ser posible en tribunales especializados $^{41}$, incluso de urgencia, y a lo largo de su celebración, se debe velar porque las víctimas tengan asistencia gratuita en todas las fases. Para evitar su victimización secundaria, deben poder estar acompañadas y representadas por especialistas y se les tiene que permitir obtener orientación y asesoramiento, incluso intérprete cuando sea preciso. Asimismo, durante el procedimiento, deben poder decidir si comparecen o no o si pueden presentar pruebas a través de medios alternativos, para no tener que encontrase cara a cara con el autor del ilícito. Su participación tiene que ser a estrictamente necesaria y se debe contar con medios físicos para que se encuentre protegida. La

38 Integración de los derechos humanos de la mujer y perspectiva de género: violencia contra la mujer. La norma de la debida diligencia como instrumento para la eliminación de la violencia contra la mujer, Informe de la Relatora sobre la violencia contra la mujer, sus causas y consecuencias, Yakin Ertürk, E/CN.4/2006/61 de 20 de enero de 2006, párr. 53 y 54.

39 Departamento de Asuntos Económicos y Sociales, División para el Adelanto de la Mujer de las Naciones Unidas, Manual de Legislación sobre la violencia contra la mujer, op. cit., p. 38.

40 Id., p. 39.

41 Abdul Aziz, Z.; Moussa, J. (dir.), Marco de la Debida Diligencia..., op. cit., p. 72. 
recopilación y presentación de pruebas será apropiada y se señalará que no son necesarias pruebas médicas o forenses para condenar a un autor por violencia; pero si éstas fueran necesarias, no podrá requerirse para que sean examinadas, el consentimiento de nadie, especialmente de una figura masculina. Asimismo, se eliminarán los elementos discriminatorios en los procedimientos judiciales sobre violencia sexual. En este sentido «la legislación debe abolir la norma de corroboración o advertencia cautelar con respecto a las demandantes en asuntos de violencia sexual, señalando que será ilegal exigir la corroboración de las pruebas de la demandante, creando una suposición de credibilidad de la demandante en asuntos de violencia sexual y afirmando que la credibilidad de la demandante en un asunto de violencia sexual será la misma que la credibilidad de una parte demandante en cualquier otro procedimiento penal», por lo que se impedirá que se valore el historial sexual de la demandante en cualquier tipo de procedimiento ${ }^{42}$.

Las órdenes de protección se extenderán a todas las víctimas, y se contendrán las siguientes medidas: «ordenar al demandado/autor del acto violento permanecer a una distancia concreta de la demandante y sus hijos (y otras personas en su caso) y de los lugares que frecuenten; ordenar al acusado que ayude financieramente a la demandante, incluido el pago de facturas médicas, honorarios de asesores o gastos de refugios, compensaciones monetarias y, además, en casos de violencia doméstica, para préstamos hipotecarios, alquileres, seguros, pensiones alimenticias y apoyo a los hijos; prohibir al demandado ponerse en contacto con la demandante o hacerlo con una tercera parte; disuadir al demandado de causar más violencia a la demandante, sus dependientes, otros familiares y personas pertinentes; prohibir que el demandado adquiera, utilice o posea un arma de fuego o cualquier otra arma que especifique el tribunal; exigir que el demandado sea objeto de seguimiento electrónico; dar instrucciones al demandado en casos de violencia doméstica para que abandone el hogar familiar, sin emitir en ningún caso dictamen sobre la propiedad de dicho inmueble y/o que dé un medio de transporte u otros efectos personales esenciales a la demandante; la emisión de órdenes de protección tanto en procedimientos penales como civiles; y que las autoridades no hagan salir a la demandante del hogar en contra de su voluntad» ${ }^{43}$.

\footnotetext{
42 Departamento de Asuntos Económicos y Sociales, División para el Adelanto de la Mujer de las Naciones Unidas, Manual de Legislación sobre la violencia contra la mujer, op. cit., pp. 40 y ss.

43 Id., p. 48.
} 
Se va más allá en relación a las órdenes de emergencia, en virtud de las cuales y en situaciones de riesgo inminente de violencia, se da autoridad a la policía para ordenar el abandono del hogar del autor del ilícito, así como la obligación de mantenerse alejado de la víctima, teniendo este procedimiento lugar con carácter ex parte, sin audiencia, y teniendo como única prioridad la seguridad de la víctima ${ }^{44}$.

Y para que estas órdenes de protección se concedan, bastará con el testimonio directo de la demandante, no siendo necesarias otras pruebas independientes ${ }^{45}$.

Una cuestión especialmente sensible se remite a la custodia de los hijos en procedimientos en los que existan órdenes de protección. En ellos, se presumirá en contra de la concesión de la custodia al autor del ilícito, se establecerá la presunción en contra del derecho de visita si ésta no es supervisada, se exigirá que antes de la concesión de la visita supervisada hayan transcurrido tres meses desde el acto violento y que el autor haya cesado en la violencia y participe en un programa de tratamiento. Finalmente, no se concederán derechos de visita en contra de la voluntad del niño ${ }^{46}$, y todo ello porque los maltratadores han venido utilizando la custodia o las visitas a los hijos e hijas para perpetuar el comportamiento violento.

La tercera fase alude al castigo o condena, que será proporcional a los delitos cometidos, y es que suelen ser desproporcionados, en tanto que son minimizados. Los casos más extremos son aquellos en los que la legislación contempla atenuantes y eximentes en el caso de delitos de honor, lo que constituye una práctica completamente contraria al estándar de debida diligencia, al igual que la exculpación del delincuente si contrae matrimonio con la víctima, su excarcelación si tienen un hijo, o que la condena se vea reducida si la víctima de violencia es una trabajadora sexual o una persona cuya conducta sexual se cuestione ${ }^{47}$. Es más, es preciso que la condena se agrave en el caso de delitos reiterativos o agravados de violencia doméstica ${ }^{48}$.

La condena se fijará con el objeto de prevenir la reincidencia, promover la rehabilitación de los delincuentes y en disuadir a terceros. De no hacerlo, se

44 Id., p. 49.

45 Id., p. 51.

46 Id., p. 52.

47 Id., p. 54.

48 Id., p. 55. 
fomenta la impunidad y se normaliza la violencia. Pero tampoco las sentencias demasiado duras son útiles, porque pueden condicionar a los agentes encargados de investigar a no seguir con el caso $^{49}$.

\subsection{Reparación}

Señalaba la Relatora Ertürk que la reparación es probablemente la parte menos desarrollada ${ }^{50}$, a pesar de ser un elemento esencial, sobre todo porque tiene por objeto satisfacer las necesidades de las víctimas, de cara a intentar eliminar o, si no es posible, mitigar, los daños sufridos por la víctima de violencia.

La reparación adopta dos formas para hacerse efectiva: de forma procedimental y sustantiva. La primera consiste en recurrir a un proceso a través del cual los órganos judiciales o administrativos evalúan las solicitudes de las víctimas. Desde el punto de vista sustantivo, estarán los resultados de estos procesos y las reparaciones que en ellos se acuerden. Con todo, si bien el derecho internacional ha venido reconociendo el derecho a la reparación, ya sea en las relaciones entre Estados tras la comisión de un ilícito, y también en el derecho internacional de los derechos humanos y en el derecho internacional humanitario ${ }^{51}$, su contenido no está claro. De hecho, como indica la Relatora «al referirse a la indemnización a raíz de la violación de algún derecho, todos los tratados de derechos humanos emplean fórmulas un tanto vagas [...], los organismos internacionales de derechos humanos competentes para recibir reclamaciones suelen limitarse a establecer los hechos y a emitir dictámenes declaratorios o, en el mejor de los casos, a recomendar que se resarza a los

49 Abdul AzIZ, Z.; Moussa, J. (dir.), Marco de la Debida Diligencia..., op. cit., p. 80.

$50 \ll$ Se dispone de pocos datos acerca de la obligación del Estado de proporcionar reparaciones adecuadas por actos de violencia contra la mujer. Algunos Estados mencionaron que las mujeres pueden obtener indemnizaciones mediante fondos para víctimas de esos delitos o mediante procedimientos civiles, pero, aparte de eso, ese aspecto de la debida diligencia sigue estando muy poco desarrollado.» Integración de los derechos humanos de la mujer y perspectiva de género: violencia contra la mujer. La norma de la debida diligencia como instrumento para la eliminación de la violencia contra la mujer, Informe de la Relatora sobre la violencia contra la mujer, sus causas y consecuencias, Yakin Ertürk, E/CN.4/2006/61 de 20 de enero de 2006, párr. 55.

51 Informe de la Relatora Especial sobre violencia contra la mujer, sus causas y consecuencias, Rashida Manjoo, A/HRC/14/22 de 23 de abril de 2010, párr. 13. 
demandantes con una indemnización sin monto preciso» ${ }^{52}$, cosa que sin embargo sí se hace en los tribunales regionales. Pueden servir para clarificar el panorama los Principios y directrices básicos sobre el derecho de las víctimas de violaciones manifiestas de las normas internacionales de derechos humanos y de violaciones graves del derecho internacional bumanitario a interponer recursos y obtener reparaciones $^{53}$. Según estos principios, la reparación debe ser proporcional y puede consistir en la restitución, la indemnización, la rehabilitación, la satisfacción y las garantías de no repetición ${ }^{54}$.

No obstante, todavía queda un largo camino por recorrer en este ámbito, y muestra de ello es el tratamiento decepcionante de la cuestión por parte del Tribunal Europeo de Derechos Humanos en el asunto Opuz contra Turquía, donde si bien sí se reconoció que Turquía había violado sus obligaciones al no respetar el estándar de debida diligencia para proteger a las mujeres contra la violencia doméstica, considerándolo una forma de discriminación de conformidad con el Convenio de Roma ${ }^{55}$, y concedió una indemnización en concep-

Id., párr. 14.

53 Comisión de Derechos Humanos, Res. 2005/35, E/CN.4/2005/L.48. Informe de la Relatora Especial sobre violencia contra la mujer, sus causas y consecuencias, Rashida Manjoo, A/ HRC/14/22 de 23 de abril de 2010, párr. 15.

54 Id., párr. 17.

La práctica desarrollada por los Estados ha dado lugar a diferentes formas de reparar:

1. «restitución o medidas para restaurar a las víctimas a su situación original antes de la violación, incluyendo la restauración de la libertad, los derechos humanos, el empleo, la identidad, la vida familiar y la ciudadanía, el regreso al lugar de residencia y la devolución de los bienes propios;

2. una indemnización por cualquier daño evaluable económicamente, de forma apropiada y proporcional a la gravedad de la violación, incluyendo daño físico o mental, pérdida de oportunidades de empleo, educación, prestaciones sociales y los daños materiales y morales;

3. la rehabilitación, incluyendo la atención médica y psicológica, así como los servicios jurídicos y sociales;

4. medidas de satisfacción que incluyen, entre otras, la verificación de los hechos y la revelación pública y completa de la verdad, la determinación del paradero de los desaparecidos, disculpas públicas, sanciones judiciales o administrativas a los responsables de las violaciones, conmemoraciones y homenajes a las víctimas; $y$

5. las garantías de no repetición, incluyendo medidas que contribuyan a la prevención, como asegurar el control civil efectivo de las fuerzas militares y de seguridad, la protección de los derechos humanos, así como la revisión y reforma de las leyes que contribuyen a, o permiten, violaciones graves del derecho internacional de los derechos humanos». ABDUL AZIZ, Z.; Moussa, J. (dir.), Marco de la Debida Diligencia..., op. cit., p. 86.

55 Tribunal Europeo de Derechos Humanos, caso Opuz contra Turquía, Demanda no. 22401/02, párr. 143 y 146 
to de daños no materiales, desestimó la indemnización por daños materiales y no procedió a la compensación por daños psicológicos ni previó otras formas de reparación ni garantías de no repetición ${ }^{56}$.

\section{ESPAÑA Y LA DEBIDA DILIGENCIA EN MATERIA DE VIOLENCIA CONTRA LAS MUJERES}

España es parte de la Convención para la Eliminación de todas las formas de Discriminación contra la Mujer ${ }^{57}$ y del Protocolo Facultativo de la Convención sobre la Eliminación de todas las formas de Discriminación contra la Mujer ${ }^{58}$, por lo que asume las obligaciones contenidas en la Convención, así como la supervisión periódica, al tiempo que las comunicaciones individuales, éstas en el marco del Protocolo. El consentimiento en obligarse por estos instrumentos resulta de utilidad a la hora de determinar si España cumple o no con sus obligaciones, más concretamente, en relación al respeto del estándar de debida diligencia.

La última vez que España presentó su informe ante el Comité de la CEDAW fue en 2015. En él, nuestro país aludía a su compromiso contra la violencia de género, a la LO 1/2004 de 28 de diciembre, de Medidas de Protección Integral contra la Violencia de Género ${ }^{59}$, así como a las medidas adoptadas, como el Observatorio Estatal de Violencia contra la Mujer, el sistema de seguimiento integral en los casos de violencia de género (Sistema VioGén), la Estrategia Nacional para la erradicación de la violencia contra la mujer (2014-2016), así como iniciativas de capacitación para personal sanitario, policial, de educación, y en el ámbito judicial, la puesta en funcionamiento de la línea de atención telefónica 016, la creación de indicadores y elaboración de encuestas, así como la

56 Id., Fallo, párr. 8 y 9.

Vid., Comisión de Derechos Humanos, Res. 2005/35, E/CN.4/2005/L.48. Informe de la Relatora Especial sobre violencia contra la mujer, sus causas y consecuencias, Rashida Manjoo, A/ HRC/14/22 de 23 de abril de 2010, párr. 80 y 81.

57 Instrumento de Ratificación de 16 de diciembre de 1983 de la Convención sobre la eliminación de todas las formas de discriminación contra la mujer, hecha en Nueva York el 18 de diciembre de 1979, BOE $\mathrm{n}^{\circ}$ 69, de 21 de marzo de 1984, páginas 7715 a 7720.

58 Instrumento de Ratificación de del Protocolo Facultativo de la Convención sobre la eliminación de todas las formas de discriminación contra la mujer, hecho en Nueva York el 6 de octubre de 1999, BOE nº 190, de 9 de agosto de 2001, páginas 29707 a 29710.

59 Comité para la Eliminación de la Discriminación contra la Mujer, Examen de los informes presentados por los Estados partes en virtud del artículo 18 de la Convención. España, CEDAW/C/ ESP/7-8 de 17 de diciembre de 2014, párr. 312. 
labor desarrollada en el marco autonómico. Si bien puede considerarse que se han implementado medidas que pueden ser útiles para cubrir determinados aspectos de la prevención sistémica, hay cuestiones que siguen fallando y permiten concluir que España no cumple con el estándar. Veámoslo.

\subsection{España y la debida diligencia sistémica}

En su informe final, el Comité de la CEDAW es sumamente crítico con España al considerar que todavía queda mucho camino por recorrer para cumplir con sus obligaciones. En este sentido, «el Comité está alarmado por la prevalencia en el Estado parte de la violencia contra la mujer, incluida la violencia sexual, y por el alto porcentaje de mujeres que han muerto como resultado de la violencia de género en las relaciones de pareja ${ }^{60} \mathrm{y}$ es que la LO 1/2004 no abarca otras formas de violencia más allá de la que se ejerce en el ámbito familiar, el número de niños asesinados en las visitas a sus padres es alarmante y como consecuencia de la crisis, las medidas de austeridad han afectado muy negativamente a los servicios de protección, como las casas de acogida y los refugios, donde además, la disponibilidad es muy limitada. En consecuencia, y en consonancia con la Observación General n ${ }^{\circ} 19$, se insta a que España adopte determinadas medidas para satisfacer el estándar de debida diligencia.

Por lo que atañe a la prevención, España debe revisar la legislación, incluyendo otras formas de violencia, más allá de la ejercida en el ámbito doméstico, como la que ejercen los cuidadores, la policía, o en centros de trabajo y escuelas. Además, es imprescindible promover la capacitación obligatoria para jueces, fiscales y policía sobre la CEDAW y el Protocolo, se deben establecer medidas integrales para combatir la violencia y es preciso que se recopilen datos estadísticos desglosados ${ }^{61}$. En relación a la protección, España debe garantizar el acceso a medios inmediatos de protección, y proporcionar asistencia y protección adecuada a las mujeres víctimas, siendo especialmente problemática la situación de las casas de acogida que han sufrido la política de austeridad en el contexto de la crisis financiera. Es particularmente alarmante la falta de

\footnotetext{
60 Comité para la Eliminación de la Discriminación contra la Mujer, Observaciones finales sobre los informes periódicos séptimo y octavo combinados de España, CEDAW/C/ESP/CO/7-8 de 29 de julio de 2017, párr. 20.

${ }^{61}$ Id., párr. 21 a) b) c) y f).
} 
acceso a estos centros de refugio en el ámbito rural ${ }^{62}$. El marco español sobre la investigación, el juzgamiento y el castigo también presenta deficiencias. Así, es imprescindible que se promueva la capacitación obligatoria, ya aludida en la prevención, hay que garantizar que los autores sean enjuiciados y castigados adecuadamente y se tiene que alentar a las mujeres a denunciar, eliminando el riesgo de estigmatización de las víctimas ${ }^{63}$. Finalmente, las medidas de reparación deben ser garantizadas ${ }^{64}$.

\subsection{España y la debida diligencia individual: el asunto Ángela González Carreño contra España}

Pero si existe un asunto que pone de manifiesto la falta de respeto de España en relación con la debida diligencia en toda su crudeza, éste es el asunto de Ángela González Carreño contra España. El presente asunto versa acerca de cómo nuestro Estado no ha sido capaz de proteger los derechos de Ángela González Carreño y de su hija Andrea, siendo éste uno de los asuntos que mayor impacto han causado en la opinión pública española en los últimos años.

Brevemente se aludirá a los antecedentes del caso para después entrar en el análisis de la mano del Comité de la CEDAW. Ángela González Carreño era víctima de violencia de género en el ámbito familiar, violencia ejercida por su marido. En 1999 la víctima abandonó el hogar familiar y denunció los hechos a la Guardia Civil y en el Juzgado correspondiente. Al poco tiempo solicitó la separación provisional y que su hija Andrea, de tres años, quedara bajo su guardia y custodia. Las visitas, solicitó, debían ser limitadas y estar vigiladas. Además, renunció al uso del domicilio familiar, lo cual fue ordenado por el juez unos meses más tarde. A pesar de ello, el acoso y el maltrato por parte de su exmarido continuó. La señora González Carreño denunció en repetidas ocasiones este maltrato, y solicitó órdenes de alejamiento, así como una pensión de alimentos. Su difícil situación económica, le condujo a reclamar el uso del domicilio familiar, dado que ella tenía a su cargo la guardia y custodia de la niña. El maltratador sólo fue condenado una vez, por vejaciones, a una multa

62 Id., párr. 21 c) y e).

63 Id., párr. 21 b) y d).

64 Id., párr. 21c). 
de 45 euros. Las órdenes de alejamiento fueron sistemáticamente violadas. Y a pesar de que las visitas vigiladas afectaban negativamente a la niña, en 2002 se autorizaron sin vigilancia. En una de estas visitas, el 24 de abril de 2003, su padre asesinó a Andrea y a continuación se suicidó.

Un año después, Ángela González Carreño presentó una reclamación de responsabilidad patrimonial al Estado por funcionamiento anormal de la Administración de Justicia, acusándola de negligente, y solicitando una indemnización como forma de reparación. El Ministerio rechazó la reclamación. Se presentó recurso de reposición, siendo también rechazado, ante lo cual se interpuso recurso contencioso administrativo ante la Audiencia Nacional, también rechazado, y recurso de casación ante el Tribunal Supremo, desestimado. Finalmente se acude en amparo ante el Tribunal Constitucional, que también rechaza el recurso ${ }^{65}$.

Posteriormente el caso llega al Comité de la CEDAW, que admite la comunicación individual y pasa a conocer del fondo del asunto, para dilucidar si existe «responsabilidad del Estado por no haber cumplido con su deber de diligencia en relación con los hechos que llevaron al asesinato de la hija de la autora ${ }^{66}$, analizando, pues, si las autoridades españolas aplicaron correctamente los principios relativos al estándar de debida diligencia, adoptando «medidas razonables con miras a proteger a la autora y su hija de posibles riesgos en una situación de violencia doméstica continuada ${ }^{67}$.

En primer lugar, se constata la ausencia de medidas de prevención. El argumento fundamental de España es que no era posible prever que existiera riesgo para la menor, afirmación que no es compartida por el Comité, dada la situación de violencia existente, el incumplimiento de las órdenes de alejamiento, y los informes tanto de los servicios sociales como psicológicos, o el que no cumpliera con la pensión de alimentos ${ }^{68}$. Además, el que se permitieran las visitas no vigiladas tenía por objeto «normalizar las relaciones entre padre e hija», y no valoraban «un interés de las autoridades por evaluar en todos sus aspectos los beneficios o perjuicios para la menor», todo lo cual «obedece a una concepción estereotipada del derecho de visita basado en la igualdad

65 Véanse los antecedentes del caso en Comité para la Eliminación de la Discriminación contra la Mujer, Comunicación no 47/2012, CEDAW/C/58/D/47/2012 de 15 de agosto de 2014, párr. 2.1 y ss.

66 Id., párr. 9.2.

67 Id., párr. 9.2.

68 Id. párr. 9.3 . 
formal que, en el presente caso, otorgó claras ventajas al padre a pesar de su conducta abusiva y minimizó la situación de madre e hija como víctimas de violencia, colocándoles en una situación de vulnerabilidad». Y es que en casos de custodia y derechos de visita debe primer el interés superior del menor, teniendo siempre en cuenta la existencia de un contexto de violencia doméstica $^{69}$. En consecuencia, tampoco se protegió ni a la madre ni a la hija. Menos aún la investigación, porque a pesar de que España «ha adoptado un modelo amplio para hacer frente a la violencia doméstica [...], para que la mujer víctima de violencia doméstica disfrute de la realización práctica del principio de no discriminación e igualdad sustantiva, y de sus derechos humanos y libertades fundamentales, la voluntad política expresada [...] debe contar con el apoyo de agentes estatales que respeten las obligaciones de diligencia debida del Estado parte. Estas obligaciones incluyen la obligación de investigar la existencia de fallos, negligencias u omisiones por parte de los poderes públicos que puedan haber ocasionado una situación de desprotección de las víctimas. El Comité considera que, en el presente caso, esta obligación no se cumplió» ${ }^{70}$. Además, «la ausencia de medidas reparatorias constituye una violación» de la Convención $^{71}$. En consecuencia, el Comité otorga una reparación adecuada a la víctima y una indemnización «integral y proporcional», exige al Estado que lleve a cabo una investigación «exhaustiva e imparcial». Y contempla medidas que debe implementar nuestro país en el marco de la debida diligencia sistémica, como son el que se desarrollen actuaciones «adecuadas y efectivas para que los antecedentes de violencia domésticas sean tenidos en cuenta en el momento de estipular los derechos de custodia y visita relativos a los hijos, y para que el ejercicio de los derechos de visita o custodia no ponga en peligro la seguridad de las víctimas de violencia, incluidos los hijos»; y refuerce «el marco legal» para asegurar el cumplimiento del estándar de debida diligencia, dando formación a jueces y otro personal ${ }^{72}$.

Si bien desde el momento en el que se cometió la violación, el marco jurídico español se ha reforzado, España se mostró en un primer momento dispuesta a cumplir con la decisión del Comité, y ello a pesar de haber aceptado su jurisdicción. Ángela González Carreño presentó un recurso extraordinario de revisión contra la Resolución del Ministerio de Justicia, que fue rechazado

\footnotetext{
69 Id., párr. 9.4.

70 Id., párr. 9.9.

71 Id., párr. 9.8 .

72 Id., párr. 11.
} 
por el Consejo de Estado en un dictamen en el que afirma que los informes de los Comités de Naciones Unidas no son vinculantes ni pueden considerarse como una interpretación auténtica del tratado ${ }^{73}$. Lamentablemente no existe en la legislación española un procedimiento que permita ejecutar los dictámenes de los órganos de Naciones Unidas, cuestión que hasta fechas muy recientes también ocurría en relación a las sentencias del Tribunal Europeo de Derechos Humanos $^{74}$, por lo que en definitiva nos sitúa ante una cuestión de voluntad política, en cuya virtud se llevara a cabo una modificación normativa que diera eficacia a las decisiones de estos órganos de supervisión, lo cual tendría toda la lógica, en tanto que si el Estado consiente en obligarse por un tratado y acepta su jurisdicción, no tiene sentido que se rechace el cumplimiento de sus decisiones $^{75}$. Parece que este razonamiento ha sido finalmente seguido por el Tribunal Supremo en la STS 1263/2018 de 17 de julio, en la que, por fin, se reconoce la responsabilidad del Estado en el presente asunto. $\mathrm{Al}$ margen de las consideraciones particulares sobre el caso de estudio, nos hallamos, como ha señalado Gutiérrez Espada, ante un punto de inflexión en relación al cumplimiento de las decisiones de los órganos convencionales de protección de los derechos humanos $^{76}$.

Pero volviendo al tema que nos ocupa, la situación es preocupante, en tanto que conduce al Comité de la CEDAW a manifestar su preocupación por la «falta de comprensión por el Estado [español] de su obligación de diligencia debida al Grupo de Trabajo sobre la cuestión de la discriminación contra la mujer en la legislación y en la práctica a criticar a España en términos muy

73 Dictamen 218/2015 de 11 de junio de 2015

74 Tras la Sentencia Del Río Prada contra España se procedió a la introducción del artículo 5 bis en la LOPJ contemplando un supuesto de revisión. Véase Simarro Pedreira, M., «El nuevo motivo de recurso de revisión (especial referencia al orden jurisdiccional penal», Revista furídica de la Universidad de León, $\mathrm{n}^{\circ}$ 3, 2016, p. 27 y ss.

75 Lousada Arochena, J.F., «El caso González Carreño contra España», Aequalitas, nº 37, 2015, p. 15, Fernández Nieto, J., «Retos y mitos de la LO 1/2004 de Violencia de Género, y reforma del Código Penal: Hacia una política criminal en violencia de género en cumplimiento del principio internacional de 'diligencia debida'», Elderecho.com, 10 de noviembre de 2015, disponible en http://www.elderecho.com/tribuna/penal/violencia-genero-mediacion-victimizacion-justicia-restaurativa-diligencia-indebida-politica-criminal_11_882430001.html [consultado: 09/05/2018], y ROMÁN MARTíN, L., La protección jurisdiccional de las víctimas de violencia de género desde la perspectiva constitucional, Tesis Doctoral, Universidad Rovira i Virgili, 2016, p. 46 y ss.

76 GutiérRez Espada, C., «La aplicación en España de los dictámenes de los comités internacionales: la STS 1263/2018, un importante punto de inflexión», Cuadernos de Derecho Transnacional, 2018, vol. 10-2, p. 836 y ss. 


\section{duros $^{77}$, y a ONGs de derechos humanos a reclamar el cumplimiento de las obligaciones $^{78}$.}

$77 \ll 71$. En el trágico caso González, en que un padre maltratador asesinó a su hija y luego se quitó la vida durante una visita no vigilada, el Comité para la Eliminación de la Discriminación contra la Mujer consideró que las autoridades del Estado, al decidir el establecimiento de un régimen de visitas no vigilado sin tener debidamente en cuenta los antecedentes de violencia doméstica, fallaron en su obligación de ejercer la debida vigilancia prevista en la Convención (CEDAW/C/58/D/47/2012, párr. 9.7). En su dictamen, el Comité formuló recomendaciones al Estado, en particular para que otorgara a la madre una reparación adecuada. Se refirió a la necesidad de tener en cuenta los antecedentes de violencia doméstica en el momento de estipular los derechos de visita para que su ejercicio no pusiera en peligro la seguridad de la mujer o los hijos, y de proporcionar formación obligatoria a los jueces y el personal administrativo sobre la violencia doméstica que incluya formación acerca de los estereotipos de género.

72. El Grupo de Trabajo considera preocupante que, en el caso mencionado, el Gobierno no haya hecho caso de las claras recomendaciones formuladas por el Comité; de hecho, su respuesta al Comité parece indicar que no ha comprendido la responsabilidad del Estado de ejercer la debida vigilancia. Es más, numerosos interesados informaron al Grupo de Trabajo durante su visita de que los funcionarios encargados de hacer cumplir la ley, incluidos los trabajadores sociales, la policía y los jueces, seguían sin dar el debido peso a las pruebas presentadas por mujeres sobre la existencia de un riesgo de violencia contra ellas y sus hijos debido a la persistencia de los estereotipos negativos de la mujer y a la insuficiente eficacia de los programas de fomento de la capacidad con una perspectiva de género. Las mujeres víctimas siguen siendo desacreditadas y calificadas de manipuladoras y a menudo son acusadas de presentar denuncias falsas. Ello puede afectar a la emisión de órdenes de protección, que se acuerdan en el $59 \%$ de los casos en que se solicitan a los tribunales. Esta cifra refleja una notable disminución del número de órdenes de protección dictadas desde 2008, año en que ese porcentaje fue del $73,3 \%$.

Diversos interesados informaron también al Grupo de Trabajo de que a menudo se interpretaba que el interés superior del niño requería una normalización de la relación con el padre, y que con frecuencia se concedían derechos de visita y custodia a los padres pese a la existencia de pruebas de que habían cometido actos de violencia doméstica. De conformidad con el artículo 66 de la Ley Orgánica n. ${ }^{\circ} 1 / 2004$, el juez podrá ordenar la suspensión de visitas del inculpado por violencia de género a sus descendientes. El Gobierno afirma que la normativa tiene por objeto velar por que, en caso de condena firme por violencia doméstica, el progenitor no tenga ningún derecho de visita, relación o comunicación hasta la extinción de su responsabilidad penal y prevé que, si se le conceden excepcionalmente tales derechos, los jueces deben adoptar las medidas necesarias para garantizar la seguridad, integridad y recuperación del niño y del otro progenitor. El Grupo de Trabajo tiene conocimiento de la iniciativa legislativa en curso en relación con la regulación de la tutela, visita y custodia en lo que respecta a los maltratadores acusados y condenados. Fue informado por el Gobierno de que la ley restringiría la potestad judicial para permitir que padres acusados o condenados por violencia doméstica mantuviesen contacto no supervisado con sus hijos. El Grupo de Trabajo pide encarecidamente que la reforma legislativa sea introducida tan pronto como sea posible, y que incluya las garantías prometidas en materia de protección del niño frente al riesgo de violencia.

74. Asimismo, según numerosos interesados, se sigue utilizando el llamado «síndrome de alienación parental» para retirar la custodia de los hijos a la madre y concedérsela al padre acusado de violencia doméstica que solicita la custodia por esa razón, en muchos casos con el apoyo de 


\section{REFLEXIÓN FINAL}

La violencia contra la mujer es, probablemente, uno de los agujeros negros más importantes en relación al cumplimiento de los derechos humanos en nuestro país, junto a la situación de los migrantes en situación de irregularidad y las condiciones establecidas para solicitar asilo y su concesión. Ahora bien, la violencia contra la mujer es un problema transversal, que puede afectar a la mitad de la población del país. Este hecho debería llevar a los poderes públicos a abordar la cuestión con decisión, siguiendo los estándares internacionales en la materia, con el objeto de brindar la mayor protección posible a las mujeres víctimas, así como a sus familiares. Los instrumentos internacionales existen, y han dado lugar a un estándar que permite al Estado dotarse de herramientas para combatir esta lacra. El estándar de debida diligencia resulta, en consecuencia, de mucha utilidad para establecer el camino a seguir. Ahora bien, en el caso español este estándar no se respeta, ni a nivel sistémico ni a nivel individual. Las causas de esta situación probablemente haya que buscarlas en la preeminencia de una sociedad patriarcal en la que los estereotipos aún están fuertemente arraigados y resulta muy complejo acabar con ellos. Ahora bien, no se trata de que el Estado quiera o no acometer esta labor. Es que está obligado a hacerlo. Y ello en virtud tanto de las normas y decisiones internacionales, como del propio texto constitucional, como es el artículo 10.2 y la relevancia que otorga a los tratados internacionales sobre derechos humanos de los que España es parte. El Pacto de Estado contra la violencia de género,

una organización de defensa de los derechos de los padres. Según esos interlocutores, el «síndrome de alienación parental» sigue siendo una enfermedad reconocida, a pesar del dictamen emitido en 2013 por el Consejo General del Poder Judicial que, en su Guía de Criterios rechaza la validez de esta teoría pseudocientífica y se opone firmemente a su uso en los casos de violencia basada en el género.

75. El Grupo de Trabajo no puede dejar de expresar su preocupación por el hecho de que las lecciones del caso mencionado más arriba no se hayan incorporado plenamente en la legislación y en la práctica. Urge la necesidad de que todas las recomendaciones del Comité para la Eliminación de la Discriminación contra la Mujer se apliquen plenamente y de inmediato.» Informe del Grupo de Trabajo sobre la cuestión de la discriminación contra la mujer en la legislación y en la práctica, Misión a España, A/HRC/29/40/Add.3 de 17 de junio de 2015, párr. 71 y ss.

78 Amnistía Internacional, Naciones Unidas, una vez más, recuerda a España que tiene que cumplir con las recomendaciones internacionales, 28 de julio de 2015. Disponible en https:// www.es.amnesty.org/en-que-estamos/noticias/noticia/articulo/naciones-unidas-una-vezmas-recuerda-a-espana-que-tiene-que-cumplir-con-las-recomendaciones-inte/ [consultado: 09/05/2018]. 
aprobado el 28 de septiembre de 2017, constituye una oportunidad perdida, y ello a pesar de que en diferentes comparecencias se mencionó el problemático cumplimento de España del estándar de debida diligencia ${ }^{79}$.

\section{BibLIOGRAFÍA}

Abdul AzIZ, Z.; Moussa, J. (dir.), Marco de la Debida Diligencia. Marco sobre responsabilidad estatal para la eliminación de la violencia contra las mujeres, Iniciativa Internacional de Derechos Humanos, 2016.

ABRAMOVICH, V., «Responsabilidad estatal por violencia de género: comentarios sobre el caso 'Campo Algodonero' en la Corte Interamericana de Derechos Humanos», Anuario de Derechos Humanos, 2010.

FARRIOR, S., «The Due Diligence Standard and Violence against Women», Interights Bulletin, $\mathrm{n}^{\circ}$ 14, 2004.

FERNÁNDEZ NieTO, J., «Retos y mitos de la LO 1/2004 de Violencia de Género, y reforma del Código Penal: Hacia una política criminal en violencia de género en cumplimiento del principio internacional de 'diligencia debida'», Elderecho.com, 10 de noviembre de 2015, disponible en http://www.elderecho.com/tribuna/penal/violencia-genero-mediacion-victimizacion-justicia-restaurativa-diligencia-indebida-politica-criminal_11_882430001.html consultado el 9 de mayo de 2018

Goldscheid, J.; Liebowitz, D.J., «Due Diligence and Gender Violence: Parsing its Power and its Perils», Cornell International Law fournal, $\mathrm{n}^{\circ} 301,2015$.

Hasselbacher, L., «State Obligations Regarding Domestic Violence: The European Court of Human Rights, Due Diligence, and International Legal Minimus of Protection», Northwestern Fournal of International Human Rights, vol. 8, 2010.

Lousada Arochena, J.F., «El caso González Carreño contra España», Aequalitas, no 37, 2015.

MarTínez VARGaS, J.R.; Vega Barbosa, G., «La obligación estatal de prevención a la luz del corpus iuris internacional de protección de la mujer contra la violencia de género», Revista Ius et Praxis, $\mathrm{n}^{\circ}$ 2, 2013.

QuRESHI, S., «The emergence/extention of Due Diligence Standard to Assess the State Response towards Violence against Women/Domestic Violence», South Asian Studies, vol. 28, 2013.

ROMÁN MARTín, L., La protección jurisdiccional de las víctimas de violencia de género desde la perspectiva constitucional, Tesis Doctoral, Universidad Rovira i Virgili, 2016.

SIMARRO PEDREIRA, «El nuevo motivo de recurso de revisión (especial referencia al orden jurisdiccional penal», Revista furídica de la Universidad de León, $\mathrm{n}^{\circ}$ 3, 2016.

79 Pero éste no se ha incluido, lo que ha conducido al Grupo Parlamentario Socialista a presentar un voto particular. Informe de la subcomisión creada para la elaboración de un Pacto de Estado en materia de violencia de género, BOCG, n 199 de 3 de agosto de 2017, Voto particular nº 34, p. 160 